\title{
Adrenocorticotropin/cortisol ratio - a marker of psoriasis severity
}

\author{
Radosław Pietrzak', Paweł Rykowski ${ }^{1}$, Anna Pasierb' ${ }^{1}$, Zbigniew Bartoszewicz², Małgorzata Stakun ${ }^{1}$, \\ Małgorzata Rykowska ${ }^{1}$, Katarzyna Karoń ${ }^{1}$, Lidia Rudnicka ${ }^{1}$, Joanna Czuwara ${ }^{1}$
}

${ }^{1}$ Department of Dermatology, Medical University of Warsaw, Warsaw, Poland

${ }^{2}$ Department of Internal Medicine and Endocrinology, Medical University of Warsaw, Warsaw, Poland

\author{
Adv Dermatol Allergol 2020; XXXVII (5): 746-750
}

DOI: https://doi.org/10.5114/ada.2019.83975

\begin{abstract}
Introduction: The hypothalamic-pituitary-adrenal (HPA) axis plays a crucial role in systemic homeostasis and hormonal regulation of metabolic and immune functions. A similar HPA axis analog exists in the skin, where it regulates inflammation, cell proliferation and differentiation. Data regarding central HPA axis dysregulation in psoriasis are interesting but so far inconclusive.

Aim: In the study we attempted to determine whether central HPA axis serum components correlate with psoriasis severity.

Material and methods: Forty-two patients (10 women and 32 men) hospitalized at the Department of Dermatology participated in the study. None of our patients received any systemic treatment. Venous blood samples were collected at 6.00 AM. The relationship between quantitative variables and psoriasis severity based on the Psoriasis Area and Severity Index (PASI) was assessed with proc logistic in SAS 9.4.

Results: The effect of adrenocorticotropin/cortisol ratio on the PASI group was OR 3.621 (95\% confidence limits $1.217-10.775)$ for a 0.1 change in ratio $(p=0.02)$, meaning ACTH/cortisol ratio positively correlates with psoriasis severity. The effect of ACTH and cortisol on the PASI group was not statistically significant, with $p$-values of 0.30 and 0.23 respectively. Other inflammatory markers such as high-sensitivity C-reactive protein, neutrophils level, LDL, and total cholesterol did not show a significant correlation with PASI score.

Conclusions: Our results support the role of HPA axis dysfunction in the complex pathogenesis of psoriasis, showing a positive correlation between morning $\mathrm{ACTH} /$ cortisol ratio and disease severity. $\mathrm{ACTH} /$ cortisol ratio can be regarded as a new biochemical marker of psoriasis severity worth further studies.
\end{abstract}

Key words: psoriasis, Psoriasis Area and Severity Index, adrenocorticotropin, cortisol, HPA axis.

\section{Introduction}

Psoriasis is a chronic inflammatory disease affecting from $0.5 \%$ up to $4 \%$ of the world's population [1]. The disease is characterized by visible scaly, inflammatory plaques of hyperproliferative skin tissue with a significant negative influence on quality of life and induction of daily life stress and anxiety [2]. The number of patients diagnosed with psoriasis rises each year, resulting in rising cost burdens of healthcare systems worldwide [3].

The hypothalamic-pituitary-adrenal (HPA) axis plays a crucial role in systemic hormonal regulation and homeostasis, and an axis analog has been discovered in the skin. Cortisol is produced via the HPA axis with circadian rhythm and acutely in response to stress. This hormone performs essential anti-inflammatory function and regulates cell fate, differentiation, and metabolism. In the skin, cortisol is responsible for inflammation inhibition, but also regulates cell apoptosis, proliferation and keratinocyte differentiation [4]. The anti-inflammatory effect of cortisol derivatives, systemic glucocorticoids (GC), has been utilized in many autoinflammatory diseases, but with a certain caution in psoriasis [5]. Treatment with systemic GC may induce severe psoriasis exacerbation including life-threatening generalized pustular subtype in case of abrupt discontinuation [6]. In psoriatic skin there is deficient endogenous glucocorticoid signaling, with inhibition of glucocorticoid synthesis and decreased expression of GC nuclear receptors, which may underline the pathogenesis of inflammation in this disease [7].

Address for correspondence: Joanna Czuwara MD, PhD, Department of Dermatology, Medical University of Warsaw, 82 A Koszykowa St, 02-008 Warsaw, Poland, e-mail: jczuwara@yahoo.com Received: 17.02.2019, accepted: 19.03.2019. 
Therefore, GS signaling restoration in psoriasis attracts attention as a promising treatment approach.

The pathophysiology of psoriasis is very complex and multifactorial. The latest studies performed on skin biopsies and cell cultures have shown that in psoriasis the balance between the components of the cutaneous HPA axis is impaired and this can contribute to uncontrolled release of inflammatory cytokines [5, 7]. It is claimed that the systemic HPA axis can supply most tissues with glucocorticoids in abundance, but data regarding the central HPA axis in psoriasis show its dysregulation and diminished response to stress. So far data regarding the central HPA axis in psoriasis are inconclusive.

\section{Aim}

In our study we attempted to ascertain whether and how central HPA axis blood components relate to psoriasis severity.

\section{Material and methods}

Forty-two patients (10 women and 32 men) hospitalized at the Dermatology Clinic of the Medical University of Warsaw participated in the study. Characteristics of the study group are presented in Table 1 . None of our patients received any systemic treatment including biological therapy, systemic retinoids, methotrexate, cyclosporin A or glucocorticoid therapy. The group of our patients was hospitalized in our department due to deterioration of their disease and unresponsiveness to topical treatment or narrow band UVB (NB-UVB) phototherapy in order to decide which treatment would be the most appropriate based on clinical examination, laboratory results and imaging diagnostic tests.

The applied protocol was approved by the Ethics Committee of the Medical University of Warsaw with the number KB/76/2016. All patients provided informed consent before participation in the study and had the histo-

Table 1. Baseline characteristics of patients with psoriasis

\begin{tabular}{|c|c|c|c|c|c|}
\hline Variable & $\begin{array}{l}\text { Mild severity } \\
\text { group }\end{array}$ & $\begin{array}{c}\text { Moderate } \\
\text { severity group }\end{array}$ & $\begin{array}{l}\text { Severe severity } \\
\text { group }\end{array}$ & $\begin{array}{l}P \text {-value for } \\
\text { variable } \\
\text { difference }\end{array}$ & $\begin{array}{l}\text { All patients with } \\
\text { psoriasis } \\
(n=42)\end{array}$ \\
\hline Age, median (IQR) [years] & $60.5(33-64)$ & $49(46-63)$ & $45(40-59)$ & 0.364 & $49(41-61)$ \\
\hline Gender ratio, $\mathrm{M} / \mathrm{F}$ & $4 / 2$ & $15 / 8$ & $13 / 0$ & 0.053 & $32 / 10$ \\
\hline Waist circumference, median (IQR) $[\mathrm{cm}]$ & $104(101-111)$ & $106(96-116)$ & $110(92-114)$ & 0.898 & $106(92-114)$ \\
\hline WHR, median (IQR) & $1.01(0.97-1.063)$ & $1.02(0.95-1.05)$ & $1.03(0.966-1.05)$ & 0.972 & $1.018(0.95-1.05)$ \\
\hline BMI, median (IQR) $\left[\mathrm{kg} / \mathrm{m}^{2}\right]$ & & & & 0.551 & $28.8(25.3-32.5)$ \\
\hline Underweight, $n$ (\%) & $0(0)$ & $0(0)$ & $1(7.69)$ & & $1(2.38)$ \\
\hline Normal, $n(\%)$ & $1(16.67)$ & $5(21.74)$ & $2(15.38)$ & & $8(19.04)$ \\
\hline Overweight, $n$ (\%) & $4(66.67)$ & $7(30.43)$ & $4(30.77)$ & & $15(35.72)$ \\
\hline Obese class $1, n(\%)$ & $0(0)$ & $8(34.78)$ & $5(38.46)$ & & $13(30.95)$ \\
\hline Obese class $2, n(\%)$ & $1(16.67)$ & $2(8.7)$ & $0(0)$ & & $3(7.14)$ \\
\hline Obese class $3, n(\%)$ & $0(0)$ & $1(4.35)$ & $1(7.69)$ & & $2(4.76)$ \\
\hline Disease duration, median (IQR) [years] & $23.2(3.19-42.84)$ & $10.9(3.5-29.41)$ & $12.2(8.4-30.6)$ & 0.504 & $11.539(4-29.41)$ \\
\hline Comorbidities, $n(\%)$ & $5(83.33)$ & $15(65.22)$ & $9(69.23)$ & 0.694 & $29(69.04)$ \\
\hline Diabetes, $n(\%)$ & $1(30.77)$ & $4(17.39)$ & $4(30.77)$ & 0.614 & $9(21.43)$ \\
\hline Hypertension, $n(\%)$ & $4(66.67)$ & $9(39.13)$ & $7(53.85)$ & 0.419 & $20(47.62)$ \\
\hline Thyroid insufficiency (in euthyroidism), $n$ (\%) & $2(33.33)$ & $3(13.04)$ & $1(7.69)$ & 0.322 & $6(14.29)$ \\
\hline Psoriatic arthritis, $n(\%)$ & $1(16.67)$ & $4(17.39)$ & $6(46.15)$ & 0.1435 & $11(26.19)$ \\
\hline $\begin{array}{l}\text { Dermatology Life Quality Index (DLQI) and } \\
\text { how it affects the patients' lives, } n(\%) \text { : }\end{array}$ & & & & 0.374 & \\
\hline Normal & $1(16.67)$ & $1(4.35)$ & $0(0)$ & & $2(4.76)$ \\
\hline Small effect & $1(16.67)$ & $3(13.04)$ & $0(0)$ & & $4(9.52)$ \\
\hline Moderate effect & $0(0)$ & $0(0)$ & $1(7.69)$ & & $1(2.38)$ \\
\hline Very large & $1(16.67)$ & $12(52.17)$ & $6(46.15)$ & & $19(45.24)$ \\
\hline Extremely large & $3(50)$ & $7(30.43)$ & $6(46.15)$ & & $16(38.1)$ \\
\hline
\end{tabular}

WHR - waist-hip ratio, $B M I$ - body mass index, IQR - interquartile range. 
pathological confirmation of psoriasis. All the procedures followed the Helsinki Declaration and the study was approved by the local clinical research ethics committee.

Clinical assessments were performed on patients at the time of enrollment. Epidemiological data were documented using a standardized questionnaire. Collected data included age, gender, height, weight, onset of the disease, history of psoriasis treatment, comorbidities (diabetes, blood hypertension, thyroid and other hormonal dysfunctions, prostate enlargement, dyslipidemias), psoriatic arthritis, body mass index (BMI), bioimpedance components (visceral fat, muscle, total fat), the occurrence of psoriasis in the family and the Dermatology Life Quality Index (DLQI). DLQI scoring was determined as follows: $0-1=$ no effect on patient's life, $2-5=$ small effect on patient's life, 6-10 = moderate effect on patient's life, $11-20$ = very large effect on patient's life, 21-30 = extremely large effect on patient's life. In the PASI a score $<10$ points indicated mild psoriasis, 10-20 indicated moderate, $>20$ indicated severe disease. Each patient underwent the following morning basic laboratory tests: blood morphological analysis, liver enzyme concentrations, fasting morning glycemia, creatinine, high-sensitivity C-reactive protein (hsCRP), albumin, thyrotropin and lipid profile. Venous blood samples were collected using vacutainer Eppendorf EDTA tubes at 6.00 AM and were transported on ice before processing. Collected blood samples were centrifuged for $15 \mathrm{~min}$ at $2000 \mathrm{~g}$ at a temperature of $5^{\circ} \mathrm{C}$. After centrifugation, sera were stored in tubes with Com plete protease inhibitor (Roche) at minus $70^{\circ} \mathrm{C}$ until being analyzed. ELISA tests (DRG ACTH (adrenocorticotropic hormone) (EIA-3647) for ACTH, LDN MS E-5000 for cortisol) were carried out in accordance with the manufacturers' instructions. Total cortisol serum concentration was expressed in $\mathrm{ng} / \mathrm{ml}$, whereas ACTH was expressed in $\mathrm{pg} / \mathrm{ml}$.

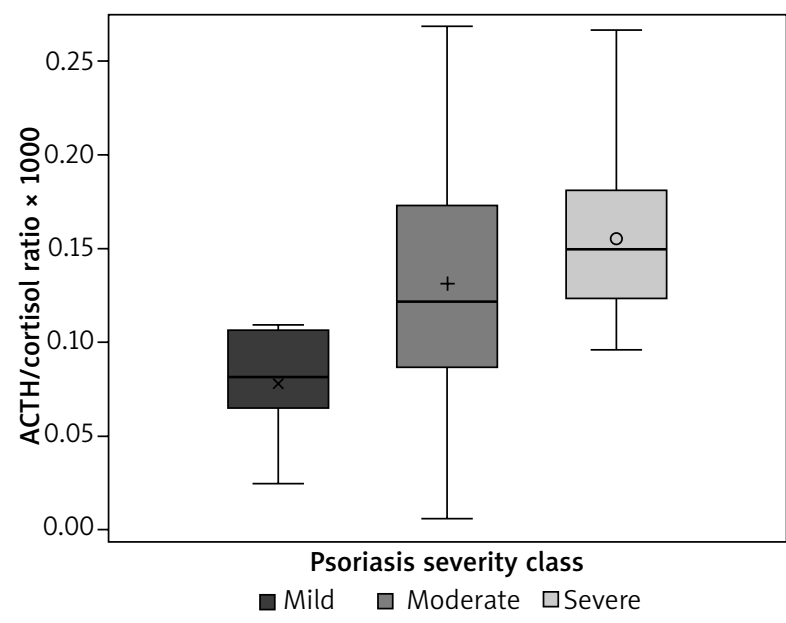

Figure 1. The graph represents predicted cumulative probabilities of adrenocorticotropin/cortisol (ACTH/COR) ratio in patients with psoriasis who have been graded with mild (PASI $<10$ ), moderate (PASI 10-20) and severe disease (PASI > 20)

\section{Statistical analysis}

Correlations were performed by Pearson correlation for parametric variables and Spearman's coefficient correlation for non-parametric variables. Conformity with the normal distribution was measured by the ShapiroWilk test. The comparisons among the groups were analyzed using the ANOVA test.

The relation between quantitative variables and psoriasis severity PASI was assessed with proc logistic in SAS 9.4. $P$-value $<0.05$ was considered statistically significant.

\section{Results}

Among 42 patients studied, 5 (12\%) had mild psoriasis (PASI < 10), 24 (57.1\%) patients moderate (PASI 10-20) and 13 (30.9\%) were diagnosed with the severe form (PASI > 20). A comparison between the groups showed no differences in variables such as age, gender, height, weight, comorbidities (diabetes, hypertension, thyroid insufficiency and other hormonal dysfunctions, prostate enlargement and other comorbidities), psoriatic arthritis, BMI, bioimpedance components (visceral fat, muscle, total fat), the occurrence of psoriasis in the family and DLQI. A detailed comparison is presented in Table 1. The majority of our patients had a very large or extremely large impact of the disease on their quality of life (in total $83.34 \%)$, confirming that psoriasis significantly affects life quality. Therefore, analysis using this indicator was not carried out in this study.

The effect of adrenocorticotropin/cortisol (ACTH/Cor) ratio on the PASI group was OR 3.621 (95\% confidence limits $1.217-10.775)$ for a 0.1 change in the ratio $(p=0.02)$ (Figure 1), meaning that $\mathrm{ACTH} /$ cortisol ratio positively and statistically significantly correlated with psoriasis severity according to PASI score evaluation at $p=0.02$. The effect of ACTH and cortisol concentrations on the PASI group was not statistically significant, with $p$ values of 0.30 and 0.23 respectively. The effect of cortisol on incidence of psoriatic arthritis (PSA) was: OR 0.987 (95\% confidence limits 0.975-0.999), $p=0.03$, meaning that PsA incidence correlated significantly with lower cortisol levels in our group of patients. The effect of ACTH/cortisol and ACTH on PSA was not statistically significant, with $p$-values of 0.50 and 0.23 respectively. Other inflammatory markers - high-sensitivity C-reactive protein, neutrophil level, low-density lipoprotein (LDL), total cholesterol vs. PASI score - attained non-statistically significant $p$ values of $0.18,0.08,0.62$ and 0.26 , respectively. ACTH/cortisol ratio significantly correlated with patients' age ( $p=0.0254, r=-0.35321)$.

\section{Discussion}

Adrenocorticotropin/cortisol ratio was not previously evaluated in patients with psoriasis, but has been reported in other medical problems, such as the Cush- 
ing syndrome diagnostic test, viral infections, sepsis and psychoneurological studies [8-11]. Our analysis showed that $\mathrm{ACTH} /$ cortisol ratio correlates with the severity of psoriasis and can be possibly utilized as a biochemical marker of disease severity.

Brunoni et al. found a positive correlation between bedtime cortisol level and psoriasis severity. In his study there was no significant psoriasis correlation with either morning cortisol or ACTH levels and these results are consistent with ours [12]. PASI and HPA axis hormones were assessed on the same day in hospitalized patients in our studies and within the same week in the study by Brunoni et al., which is important for the interpretation of the possible relationship.

Robati et al. demonstrated what cortisol levels increased significantly after anti-psoriatic treatment application consisting of conventional topical, systemic or phototherapy, not otherwise specified, when a PASI-75 response was achieved. In their observations, the cortisol levels did not differ significantly between healthy controls and study group at the baseline. This may be explained by the fact that the range of "normal" cortisol levels is broad, because it is influenced by variety of internal and external factors [13]. In our analysis we tried to adjust some of them, such as comorbidities, BMI, and results from bioimpedance, in the regression, but the small size of the group did not allow us to perform an objective evaluation.

We cannot exclude the potential influence of topical glucocorticoids on the cortisol level or ACTH/cortisol ratio, but all of our patients received such treatment. Topical glucocorticoids constitute the mainstay topical therapy in psoriasis and it is difficult to find a group of patients without it. None of our patients was on systemic treatment. Atzeni et al. in their study showed that cortisol/ACTH ratio did not differ before and after treatment with anti-TNF- $\alpha$ antibodies and etanercept in patients with psoriatic arthritis (PSA) [14]. The study also revealed that improvement was related to a higher cortisol level in comparison to other adrenal hormones, such as 17-hydroxyprogesterone and androstenedione [14]. This finding can be explained in two ways. If the cortisol/ACTH ratio did not differ after etanercept therapy, it could mean that our results are coincidental, but the group of patients in the Atzeni et al. study was much smaller and consisted of 11 patients with PSA. On the other hand, restoration of cortisol levels in comparison to other adrenal hormones supports the hypothesis that the hypothalamic-pituitary-adrenal axis is part of the pathogenesis of psoriasis, and this confirms our results.

Several studies have shown that the cortisol response to stress is diminished in psoriasis, either locally in the skin or systemically $[7,15]$. Cortisol as a key component of the HPA axis is induced by psychological stress, but psoriasis patients under higher stress influence present lower mean cortisol levels than patients who experienced lower levels of daily stressors [15]. This indicates that the HPA axis is disrupted especially in patients with psoriasis upon high levels of stressors. This finding can also explain our results of a positive correlation of $\mathrm{ACTH} /$ cortisol ratio with the severity of psoriasis, when both the disease itself and stress impact cortisol significantly with its regulation by $\mathrm{ACTH}$.

Reece et al. presented the results of $\mathrm{ACTH} /$ cortisol ratio in opioid-dependent patients. The study showed that $\mathrm{ACTH} /$ cortisol ratio negatively correlated with the age of female patients and was proposed to be an aging biomarker [10]. Regardless of the gender and different disease, these results coincide with our data showing a similar relationship, that ACTH/cortisol ratio significantly negatively correlates with the patients' age.

We should mention that our results should be interpreted in the light of their limitations. These are: the small number of patients, no control group for equalization and the lack of bedtime hormonal analysis. It would be interesting to compare ACTH and cortisol values between psoriasis patients and healthy controls to identify differences, but the same limiting factor was also present in the Brunoni et al. study with similar results to ours [12]. On the other hand, a very important requirement for the healthy control study is hospitalization, during which the assessment of HPA axis components is more reliable than randomly performed measurement in an outpatient clinic. Therefore in our study the analysis of hormonal results was carried out on hospitalized patients with several differentiating factors such as psoriasis severity, age, psoriatic arthritis and comorbidities.

\section{Conclusions}

Our results support the hypothesis of the role of HPA axis dysfunction in the pathogenesis of psoriasis. The significant correlation between adrenocorticotropin/cortisol ratio with psoriasis severity is an interesting finding, constituting one element in the HPA axis and should be treated as the basis for further studies, including daytime and bedtime blood analysis to determine whether deficient $\mathrm{ACTH} /$ cortisol regulation is constant or variable depending on the time of day.

\section{Acknowledgments}

This project was funded by grant 1M4/NM2/17 from the Medical University of Warsaw.

\section{Conflict of interest}

The authors declare no conflict of interest.

\section{References}

1. Schafer T. Epidemiology of psoriasis. Review and the German perspective. Dermatology 2006; 212: 327-37. 
2. Bozek A, Reich A. The reliability of three psoriasis assessment tools: psoriasis area and severity index, body surface area and physician global assessment. Adv Clin Exp Med 2017; 26: 851-6.

3. Brezinski EA, Dhillon JS, Armstrong AW. Economic burden of psoriasis in the United States: a systematic review. JAMA Dermatol 2015; 151: 651-8.

4. Stojadinovic O, Lee B, Vouthounis C, et al. Novel genomic effects of glucocorticoids in epidermal keratinocytes: inhibition of apoptosis, interferon-gamma pathway, and wound healing along with promotion of terminal differentiation. J Biol Chem 2007; 282: 4021-34.

5. Hannen R, Udeh-Momoh C, Upton J, et al. Dysfunctional skin-derived glucocorticoid synthesis is a pathogenic mechanism of psoriasis. I Invest Dermatol 2017; 137: 1630-7.

6. Reich A. Interleukin-17 blockade in generalized pustular psoriasis - new hope for severely ill patients. Br I Dermatol 2017; 176: $572-3$.

7. Slominski AT, Brozyna AA, Tuckey RC. Cutaneous glucocorticoidogenesis and cortisol signaling are defective in psoriasis. J Invest Dermatol 2017; 137: 1609-11.

8. Le Goaster J, Rougeot C, Tekaia F, et al. ACTH/beta-endorphins and ACTH/cortisol ratios as early biological markers in HIV infection. J Environ Pathol Toxicol Oncol 1990; 10: 322-5.

9. Selek A, Cetinarslan B, Canturk Z, et al. The utility of preoperative $\mathrm{ACTH} / \mathrm{Cortisol}$ ratio for the diagnosis and prognosis of cushing's disease. J Neurosci Rural Pract 2018; 9: 106-11.

10. Reece AS, Hulse GK. Elevation of the ACTH/cortisol ratio in female opioid dependent patients: a biomarker of aging and correlate of metabolic and immune activation. Neuro Endocrinol Lett 2016; 37: 325-36.

11. Gold JR, Divers TJ, Barton MH, et al. Plasma adrenocorticotropin, cortisol, and adrenocorticotropin/cortisol ratios in septic and normal-term foals. J Vet Intern Med 2007; 21: 791-6.

12. Brunoni AR, Santos IS, Sabbag C, et al. Psoriasis severity and hypothalamic-pituitary-adrenal axis function: results from the CALIPSO study. Braz J Med Biol Res 2014; 47: 1102-6.

13. Robati RM, Toossi P, Rahmati-Roodsari M, et al. Association of psoriasis severity with serum prolactin, thyroid hormones, and cortisol before and after treatment. Sci World I 2013; 2013: 921819.

14. Atzeni F, Sarzi-Puttini P, DePortu S, et al. In etanercepttreated psoriatic arthritis patients clinical improvement correlated with an increase of serum cortisol relative to other adrenal hormones. Clin Exp Rheumatol 2008; 26: 103-8.

15. Evers AW, Verhoeven EW, Kraaimaat FW, et al. How stress gets under the skin: cortisol and stress reactivity in psoriasis. Br J Dermatol 2010; 163: 986-91. 